\title{
On tokamak equilibria with a zero current or negative current central region
}

\author{
M.S. Chu and P.B. Parks \\ General Atomics, P.O. Box 85608, San Diego, California 92186-5608
}

(September 9, 2002)

\begin{abstract}
Several tokamak experiments have reported the development of a central region with vanishing currents (the current hole). Straightforward application of results from the work of Greene, Johnson and Weimer [Phys. Fluids, 3, 67 (1971)] on tokamak equilibrium to these plasmas leads to apparent singularities in several physical quantities including the Shafranov shift and casts doubts on the existence of this type of equilibria. In this paper, the above quoted equilibrium theory is re-examined and extended to include equilibria with a current hole. It is shown that singularities can be circumvented and that equilibria with a central current hole do satisfy the magnetohydrodynamic equilbrium condition with regular behavior for all the physical quantities and do not lead to infinitely large Shafranov shifts. Isolated equilibria with negative current in the central region could exist. But equilibria with negative currents in general do not have neighboring equilibria and thus cannot have experimental realization, i.e. no negative currents can be driven in the central region.
\end{abstract}

Typeset using REVTEX 


\section{MOTIVATION}

It was reported recently by several experimental teams ${ }^{1-3}$ that tokamaks have been observed to develop a large central current hole. The toroidal current in this region is measured to be consistent with the value zero. These central current hole states have been sustained for a large fraction of the discharge life time. Development of inductive electric fields in the experiments indicate that negative currents should have appeared in the current hole region; yet negative currents have never been observed. ${ }^{2,3}$ If the current in the central region is indeed zero, in this region there is no poloidal field and no rotational transform. It is generally understood that rotational transform provides plasma confinement and equilibrium. We are then faced with the interesting situation of joining together a region with no confinement to an external region with confinement. A straightforward application of the formulae given in the work of Greene, Johnson and Weimer ${ }^{4}$ (hereafter referred as GJW) shows that quite a few quantities such as the Shafranov shift could become singular. The question that naturally arises is, when the plasma develops current holes, is it in true equilibrium in the sense that it satisfies the force balance equation? Equilibrium with zero central current and magnetic field, and driven completely by pressure, was reported by Jensen et al. ${ }^{5}$ Stability of equilibria with current holes have been studied by Huysmans et $a l .{ }^{6}$ using a cylindrical plasma model. The plasma has been predicted to develop continuous axisymmetric resistive kink instabilities. It is well recognized that stability of a toroidal plasma is strongly affected by the toroidal effect and could be quite different from that of a cylindrical model. It was conjectured in Stratton et al. ${ }^{7}$ that no regular equilibria (with nested flux surfaces) exist with sufficiently reversed internal current. It was shown that a reconnection event could occur preventing these from forming, but no proof was given of their non-existence. No instabilities have been reported by the experimental teams. In this paper, we re-examine the work of GJW ${ }^{4}$ and show that plasma states with a current hole can satisfy

the usual magnetohydrodynamic equilibrium or force balance conditions. In particular, a central region with constant pressure and zero poloidal flux can be matched with an outside 
region with toroidal current and pressure gradient. All physical quantities, including the flux surface shapes of these equilibria, are well behaved and the Shafranov shift is finite. The boundary between the central current hole and the positive current region is a contact discontinuity. Physical quantities such as currents can have jumps across the boundary but no singularity. The equilibria also vary continuously with continuous changes in profile and boundary shapes. Thus they are experimentally realizable. We also show that equilibria with negative currents in the central region or between the current hole and an external positive current region can only be isolated equilibria, if they exist at all. These equilibria do not depend continuously on boundary and profile data and therefore they do not correspond to experimentally realizable states. We know that experimental attempts have been made by using electron cyclotron current drive (ECCD) to drive negative currents directly in the current hole region without success. ${ }^{8}$ The non-existence of this type of equilibrium might be the explanation of the non-observation of negative currents in the central region. 


\section{INCLUSION OF CURRENT HOLE EQUILIBRIA IN THE THEORY OF GREENE, JOHNSON AND WEIMER}

In this section, we follow Greene, Johnson and Weimer ${ }^{4}$ to derive the equilibrium equations that are satisfied by a tokamak and specialize to the limit of large aspect ratio. We show that their theory, if carefully re-examined, can accommodate a central current hole region. We also show that although it might be possible to find isolated equilibria with negative current in between the current hole and the positive current region, these are isolated equilibria and do not have neighboring equilibria solutions. They do not correspond to experimentally realizable states.

We start with adopting their coordinate system $(r, \theta, \phi)$. This system is connected with the cylindrical coordinate system $(X, \phi, Z)$ shown in Fig. 1 by

$$
\begin{aligned}
& X=X(r, \theta), \\
& Z=Z(r, \theta) .
\end{aligned}
$$

The magnetic field $\vec{B}$ is given by

$$
\vec{B}=R B_{0}[f(r) \vec{\nabla} \phi \times \vec{\nabla} r+g(r) \vec{\nabla} \phi]
$$

with $R$ a constant length and $B_{0}$ a constant magnetic field magnitude introduced to make $f$ and $g$ dimensionless. Since a large number of equations used in the present paper first appeared in the work of Greene, Johnson and Weimer, ${ }^{4}$ we adopt a system of double labels for the equations in the present paper. Whenever possible, the equations will be labelled not only by their proper numbers for the present paper, they will also be labelled by the numbers as they appeared in Greene, Johnson and Weimer. ${ }^{4}$ Equations used in their paper will be labelled with a "G" letter followed by the equation number in the original text. Note that if $g$ were $g(r, \theta)$, Eq. (3) would be the most general divergence-free field in which magnetic lines lie on constant $r$ surfaces. The restriction of $g$ to be a function of $r$ only causes the 
current lines to also lie on these surfaces. In our present re-examination of their paper, we assume $g(r)$ never vanishes, but $f(r)$ is allowed to vanish over a central region,

$$
f(r)=0 \quad \text { for } \quad r \leq r_{0}
$$

Since $f(r)=0$ over a region, but it is non-zero for $r>r_{0}, f(r)$ cannot be analytic to all powers in $r-r_{0}$. In the following, we will make the implicit assumption that quantitities like $f(r)$ possess sufficient number (usually two) of one sided derivatives to allow the development of the theory. The current is given by

$$
\begin{aligned}
\vec{J}= & R B_{0}\left(-g^{\prime}(r) \vec{\nabla} \phi \times \vec{\nabla} r+\frac{X}{\mathcal{J}}\left\{\frac{\partial}{\partial r} \frac{f}{\mathcal{J} X}\left[\left(\frac{\partial X}{\partial \theta}\right)^{2}+\left(\frac{\partial Z}{\partial \theta}\right)^{2}\right]\right.\right. \\
& \left.\left.-\frac{\partial}{\partial \theta} \frac{f}{\mathcal{J} X}\left[\left(\frac{\partial X}{\partial r} \frac{\partial X}{\partial \theta}\right)+\left(\frac{\partial Z}{\partial r} \frac{\partial Z}{\partial \theta}\right)\right]\right\} \vec{\nabla} \phi\right) .
\end{aligned}
$$

In Eq. (5), $\mathcal{J}$ is the Jacobian of the coordinate transformation

$$
\mathcal{J}=\frac{\partial X}{\partial \theta} \frac{\partial Z}{\partial(\epsilon r)}-\frac{\partial X}{\partial(\epsilon r)} \frac{\partial Z}{\partial \theta}
$$

In the following, we do not intend to repeat all the equations in the paper of Greene et al. We will only repeat some of the more crucial equations and display equations that need modification. Based on general physical grounds we may infer that when the magnetic fields and currents are well defined, the globally integrated fluxes are also well defined. We can examine the equations of $(\mathrm{G} 7)$ for the toroidal flux $\Psi(r)$, (G8) for the poloidal flux $\chi(r)$, (G9) for the rotational transform $\iota(r),(\mathrm{G} 10)$ for the toroidal current flux $I(r)$, (G11) for the poloidal current flux $K(r)$, and (G12) for the poloidal magnetic field $\vec{B}_{p}$ to find that all these quantities are well behaved even if there is a central current free region. Substitution of Eqs. (5) and (3) into the equilibrium equation

$$
\vec{J} \times \vec{B}=p^{\prime}(r) \vec{\nabla} r
$$

then gives

$$
\begin{aligned}
\frac{p^{\prime}}{R^{2} B_{0}^{2}}+\frac{g g^{\prime}}{X^{2}} & +\frac{f}{\mathcal{J} X}\left\{\frac{\partial}{\partial r} \frac{f}{\mathcal{J} X}\left[\left(\frac{\partial X}{\partial \theta}\right)^{2}+\left(\frac{\partial Z}{\partial \theta}\right)^{2}\right]\right. \\
- & \left.\frac{\partial}{\partial \theta} \frac{f}{\mathcal{J} X}\left(\frac{\partial X}{\partial r} \frac{\partial X}{\partial \theta}+\frac{\partial Z}{\partial r} \frac{\partial Z}{\partial \theta}\right)\right\}=0 .
\end{aligned}
$$


It is important to notice that in Eq. (8), $p, f$, and $g$ are all functions of $r$ only. To proceed, the large aspect ratio assumption $a / R \ll 1$ is invoked, where $a$ is the plasma minor radius. The standard tokamak ordering demands $f \sim p^{1 / 2} \sim a / R, g \sim 1$. The tag $\epsilon$ is assumed to be of the order of $a / R$. The equilibrium relation Eq. (8) is solved by finding the flux surface shapes and the global integral quantities in terms of the input functions of $p(r)$ and $f(r)$ and boundary data. All of this information is obtained in a series development in $\epsilon$. The expansion of the coordinates are in the form

$$
\begin{aligned}
& X=R-\epsilon r \cos \theta-\epsilon^{2} \Delta(r)+\epsilon^{3}[E(r)+P(r)] \cos \theta+\ldots, \\
& Z=\epsilon r \sin \theta+\epsilon^{3}[E(r)-P(r)] \sin \theta+\ldots
\end{aligned}
$$

with the expansion of the Jacobian as

$$
\mathcal{J}=\epsilon r+\epsilon^{2} r \Delta^{\prime} \cos \theta-\epsilon^{3}\left[(r P)^{\prime}+r^{2}\left(\frac{E}{r}\right)^{\prime} \cos 2 \theta\right]+\ldots
$$

We note that the coordinate transformation is well behaved if $\mathcal{J}$ in Eq. (11) is well behaved. This demands that the unknown functions of $\Delta(r), P(r), E(r), \ldots$ possess first order derivatives. $\Delta(r)$ is the Shafranov shift; $P(r)$ is the poloidal flux renormalization; and $E(r)$ is the ellipticity. However, the current expression given in Eq. (5) demands that although only the first order derivatives of current and pressure profiles are needed, the shape functions are required to possess finite one sided second order derivatives. The lower order terms of the quantities $g(r), f(r)$, and $p(r)$ are given by

$$
\begin{gathered}
g(r)=1+\epsilon^{2} g^{(2)}(r)+\epsilon^{4} g^{(4)}(r)+\ldots, \\
f(r)=\epsilon f^{(1)}(r)+\epsilon^{3} f^{(3)}(r)+\ldots \\
p(r)=\epsilon^{2} p^{(2)}(r)+\epsilon^{4} p^{(4)}(r)+\ldots
\end{gathered}
$$

Here, we need to be more specific about the solution of Eq. (8) we are seeking. We seek a solution of Eq. (8) in two separate regions, region (I) $r \leq r_{0}$ and region (II) $r \geq r_{0}$. In 
each region, Eq. (8) is satisfied separately. As a matter of fact, in region (I), we give the solution as $p(r)=p\left(r_{0}\right), g(r)=g\left(r_{0}\right), f(r)=0$. Obviously, the values of $p\left(r_{0}\right)$ and $g\left(r_{0}\right)$ are demanded to be continuous across the bounding surface $r_{0}$. As in GJW's original paper, we assume that the bounding shape of plasma at $r=a$ is given. The unconventional nature of the present problem is that the shape of the boundary between regions (I) and (II) is not determined a priori but only by the solution of region (II). In the following we intend to show that this solution procedure gives us robust equilibrium which depends continously on boundary and profile data with no singularity in any physical quantity.

It is straightforward to substitute Eqs. (9), (10), and (11) into the expressions for toroidal flux $\Psi(r)(\mathrm{G} 7)$, poloidal flux $\chi(r)$ (G8), the rotational transform $\iota(r)$ (G9), the toroidal current $I(r)(\mathrm{G} 10)$, and the poloidal current $K(r)(\mathrm{G} 11)$ to see that these quantities remain well defined after the coordinate transformation. This results in expressions of (G30), (G31), (G32), (G33) and (G34). We would like to mention that there are apparent singularities in expressions (G32) and $\mathrm{G}(33)$ in which the first order quantity $f^{(1)}$ appears in the denominator. The regular expressions for these quantities should have the $f^{(1)}$ outside of the brakets multiplied through. Or

$$
\iota(r)=\frac{2 \pi R}{r}\left(f^{(1)}-\epsilon^{2}\left\{\left[\frac{r^{2}}{2 R^{2}}+\frac{\left(r^{2} \Delta\right)^{\prime}}{2 R r}-\frac{(r P)^{\prime}}{r}+g^{(2)}\right] f^{(1)}-f^{(3)}\right\}+\ldots\right) .
$$

Substitution of Eqs. (9), (10), and (11) into the equilibrium relation Eq. (8) and setting the Fourier coefficients equal to zero in each order, we obtain

$$
\left[\frac{p^{(2)}}{B_{0}^{2}}+g^{(2)}\right]^{\prime}+\frac{f^{(1)}}{r}\left[r f^{(1)}\right]^{\prime}=0
$$

This is the fundamental pressure balance equation.

$$
f^{(1)^{2}} \Delta^{\prime \prime}+\left[\frac{f^{(1)^{2}}}{r}+2 f^{(1)^{\prime}} f^{(1)}\right] \Delta^{\prime}-\frac{2 f^{(1)}\left[r f^{(1)}\right]^{\prime}}{R}-\frac{f^{(1)^{2}}}{R}-\frac{2 r g^{(2)^{\prime}}}{R}=0 .
$$

This is the usual hoop force balance equation.

$$
\begin{aligned}
f^{(1)^{2}} E^{\prime \prime}+ & {\left[\frac{f^{(1)^{2}}}{r}+2 f^{(1)^{\prime}} f^{(1)}\right] E^{\prime}-\frac{3 f^{(1)^{2}}}{r^{2}} E+\frac{f^{(1)}\left(r f^{(1)}\right)^{\prime}}{2 r}\left(\frac{3 r^{2}}{R^{2}}-\frac{4 r \Delta^{\prime}}{R}+3 \Delta^{\prime 2}\right) } \\
& +f^{(1)^{2}}\left(\frac{3 r}{2 R^{2}}+\frac{\Delta^{\prime}}{2 R}-\frac{r \Delta^{\prime \prime}}{R}-\frac{3 \Delta^{\prime 2}}{2 r}+\frac{3 \Delta^{\prime} \Delta^{\prime \prime}}{2}\right)+\frac{3 r^{2} g^{(2)^{\prime}}}{2 R^{2}}=0 \quad .
\end{aligned}
$$


This is the ellipticity equation.

$$
\begin{aligned}
& f^{(1)^{2}} P^{\prime \prime}+\left[\frac{f^{(1)^{2}}}{r}+2 f^{(1)^{\prime}} f^{(1)}\right] P^{\prime}+\frac{1}{r^{2}} P f^{(1)^{2}}+\left\{\left[\frac{p^{(4)}}{B_{0}^{2}}+g^{(4)}\right]^{\prime}+g^{(2)} g^{(2)^{\prime}}+\frac{1}{r^{2}}\left[r^{2} f^{(1)} f^{(3)}\right]^{\prime}\right. \\
& \left.+\frac{3 r^{2} g^{(2)^{\prime}}}{2 R^{2}}+\frac{2 \Delta g^{(2)^{\prime}}}{R}\right\}+f^{(1)^{2}}\left(\frac{3 r}{2 R^{2}}+\frac{\Delta^{\prime}}{2 R}-\frac{r \Delta^{\prime \prime}}{R}-\frac{\Delta^{\prime 2}}{2 r}+\frac{3 \Delta^{\prime} \Delta^{\prime \prime}}{2}\right) \\
& +\frac{f^{(1)}\left[r f^{(1)}\right]^{\prime}}{2 r}\left(\frac{3 r^{2}}{R^{2}}-\frac{4 r \Delta^{\prime}}{R}+3 \Delta^{\prime 2}+\frac{4 \Delta}{R}\right)=0 .
\end{aligned}
$$

This is the flux renormalization equation. Equations for shape functions $S(r)$ which describe higher order multiple harmonic distortion of the flux surface shape and/or higher order in the large aspect ratio expansion parameter $\epsilon$ have also been examined. They can be written in the general form

$$
f^{(1)^{2}} S^{\prime \prime}+\left[\frac{f^{(1)^{2}}}{r}+2 f^{(1)^{\prime}} f^{(1)}\right] S^{\prime}+\frac{c_{0}}{r^{2}} S+L(r)=0
$$

Here, $c_{0}$ is a constant, and $L(r)$ is the source which affects the multiple harmonic distortion and depends only on the lower order shape and profile functions. It is interesting to note that actually $\Delta, E$, and $P$ also satisfy equations in the form (20).

\section{A. The Presence of a Central Current Hole}

We now examine the equations given in Eqs. (16), (17), (18), and (19) and try to solve them in the region $r_{0} \leq r \leq 1$. These equations are the same independent of the equilibrium we are seeking. We note that for regular equilibria, the solution is sought in the region $0 \leq r \leq 1$. It is well known that tokamaks have robust equilibrium solutions without the current hole. As we have discussed in the previous section, the solution in region (I) is trivial and can be connected with any solution in region (II) without difficulty: we only need to show that for equilibria with a current hole, the solutions have the same properties up to the surface $r=r_{0^{+}}$as the solutions for a regular equilibrium at $r=0^{+}$. In the original work of Greene et al., ${ }^{4}$ Eqs. (17), (18), and (19) were displayed first divided by $f^{(1)^{2}}$. Displaying them in the present form facilitates our recognition that these equations are satisfied trivially in the region $r \leq r_{0}$. The quantity $f^{(1)}$ is related to the rotational transform $\iota(r)$ Eq. (15) through 


$$
f^{(1)}(r)=\frac{r \iota^{(0)}(r)}{2 \pi R}
$$

We note that for equilibria without a current hole and with a finite rotational transform $\iota(0)$ (hereafter referred to as regular equilibria), near the magnetic axis, $f^{(1)} \sim r$. Whereas for equilibria with a current hole, for $r \geq r_{0}$, we expect

$$
f^{(1)}(r) \sim\left(r-r_{0}\right)^{n}
$$

with $n \geq 1$, if the current is at most finite at $r \geq r_{0}$. With $n=1$, there is a finite current jump at $r=r_{0}$. This means that for the case of equilibria with current hole, $f^{(1)}(r)$ does not have to go to zero at a faster rate near $r=r_{0}$ than its behavior near the magnetic axis for a regular equilibrium. This observation is crucial to all the following developments. We show that using this property, all of the equations that determine the equilibria with a current hole at $r \geq r_{0}$ are not more singular than the case near the magnetic axis of a regular equilibrium. Next we would like to study the solution of these equations. It is quite clear that we need to only examine the solution of these equations for a region $r \leq r_{0}+d$, with $d$ some finite distance. In the region $r \geq r_{0^{+}}$, we may determine the quantities $g^{(2)}$ and $\Delta$ in terms of $p^{(2)}$ and $f^{(1)}$. These functions are supposed to satisfy the boundary conditions

$$
\begin{aligned}
& p^{(2)}\left(r_{0^{+}}\right)=p^{(2)}\left(r_{0^{-}}\right), \\
& g^{(2)}\left(r_{0^{+}}\right)=g^{(2)}\left(r_{0^{-}}\right)
\end{aligned}
$$

and

$$
f^{(1)}\left(r_{0^{+}}\right)=0
$$

From Eq. (16) we obtain

$$
g^{(2)}(r)=\frac{-p^{(2)}(r)}{B_{0}^{2}}+\int_{r}^{a} \frac{f^{(1)}(r)}{r}\left[r f^{(1)}(r)\right]^{\prime} d r
$$

This equation is actually identical to that given in GJW. In order for the solution to connect smoothly across $r=r_{0}$, we verify that because of Eqs. (23), (24) and $(25), g^{(2)^{\prime}}\left(r_{0^{+}}\right)=$ 
$-\left[p^{(2)^{\prime}}\left(r_{0^{+}}\right)\right] / B_{0}^{2}$. We notice that equation Eq. (16) does not preclude $p^{(2)^{\prime}}\left(r_{0^{+}}\right)$from being finite. Next we may integrate Eq. (17) and obtain

$$
\begin{aligned}
& \Delta^{\prime}(r)=\frac{1}{R r f^{(1)^{2}}(r)} \int_{r_{0}}^{r}\left[f^{(1)^{2}}(r)-\frac{2 r p^{(2)^{\prime}}(r)}{B_{0}^{2}}\right] r d r, \\
& \Delta(r)=\frac{1}{R} \int_{r_{0}}^{r} \frac{d r}{r f^{(1)^{2}}(r)} \int_{r_{0}}^{r}\left[f^{(1)^{2}}(r)-\frac{2 r p^{(2)^{\prime}}(r)}{B_{0}^{2}}\right] r d r .
\end{aligned}
$$

From Eq. (27), we know that in order for $\Delta^{\prime}$ to be well behaved across $r=r_{0}$, then

$$
p^{(2)^{\prime}} \sim \beta_{p}\left(r-r_{0}\right)^{m}
$$

with $m \geq 2 n-1$, i.e. similar to the case close to the magnetic axis, the pressure profile is flat near the current hole. In Eq. (29), we introduced the quantity $\beta_{p}$ as a measure of the strength $p^{\prime}$ in the force balance relationship. When $m=2 n-1$, then $\Delta^{\prime}\left(r_{0^{+}}\right) \sim \beta_{p}$, $\Delta\left(r_{0^{+}}\right)=0$. Therefore although $\Delta^{\prime}\left(r_{0}\right)$ has a jump, $\Delta\left(r_{0}\right)$ connects continuously across.

We see that despite the original comment in GJW that " $\Delta(r)$ is infinite when $f(r)$ vanishes; a rotational transform is necessary for equilibrium," we have shown that a region with zero rotational transform does not preclude its connection to an equilibrium region with a finite rotational transform. In particular, the Shafranov shift $\Delta(r)$ is finite for $r \geq r_{0}$.

We may continue to examine Eqs. (18) and (19). We note that for regular equilibrium without a current hole, near the magnetic axis, we have two contributions to the singularity, one coming from the radius $r$ being 0 , the other coming from $f^{(1)}(0)$ being 0 . In the case with a current hole, near $r=r_{0}$, we have only the singularity contribution from $f^{(1)}\left(r_{0}\right)$ being 0 . In the case of current hole equilibria, the way that $f^{(1)}(r)$ approaches 0 does not have to be faster than the case when it approaches the magnetic axis for regular equilibria. Just as in the case of regular equilibria, the left hand boundary point is a singular boundary point. Therefore although this is a second order differential equation, only one boundary condition may be specified. In this case, we take it to be the value of the ellipticity at the plasma surface. Therefore, this equation is completely well behaved and its solution depends continuously on profile and boundary data. The solution of this equation gives 
us the ellipticity of the surface $r=r_{0}$. However, not all the higher derivatives of $E(r)$ can be continuous across $r=r_{0}$. For convenience, we assume that we choose the function $E(r)=E\left(r_{0}\right)$ inside of $r=r_{0}$. We have shown that the solution outside is well behaved, and seek a power series solution for $E(r)$ in $r \geq r_{0}$. The power series starts with the constant value $E\left(r_{0}\right)$. Then by substituting the power series into Eq. (18), we find that the derivative $E^{\prime}\left(r_{0}\right)$ is actually determined. Therefore, we will have a jump in $E^{\prime}\left(r_{0}\right)$ across $r_{0}$, although in the present context, this jump has no physical significance. The discussion of the other equations is similar to this. Therefore, we may solve these two equations in a similar fashion to that in GJW. Because the nature of the singularity is the same for the higher order shape functions described by Eq. (20), there should be no extra difficulty in solving them.

It is well known that regular equilibria with no current holes are well behaved near the magnetic axis. Therefore, equilibria with central hole do exist and are robust. These equilibria depend continuously on profile and boundary data and are experimentally realizable. All the physical quantities are continuous across the suface $r=r_{0}$, but the derivatives may be discontinuous. Thus the location $r=r_{0}$ is a contact discontinuity. The unconventional nature of the present situation arises from the fact that the shape of $r=r_{0}$ is a part of the solution (a free boundary).

\section{B. Negative Current in the Central Region}

It has also been reported by the experimental teams that the development of inductive electric fields in the plasma should have driven currents negative in the central region. Yet no negative currents have been observed. In this subsection we examine the possible equilibria in this case. If there are negative currents, then, inside the plasma, there will exist a radius $r_{1}$, the poloidal magnetic field reversal layer, with the total toroidal current inside being zero. At such a location, again, $f\left(r_{1}\right)=0$. We discuss the possibility of a solution of the equilibrium equation in three regions, region (I) $r \leq r_{0}$, region (II) $r \geq r_{1}$, and region (M) $r_{0} \leq r \leq r_{1}$. We notice that the solutions of regions (I) and (II) are the same as before, 
so we need to concentrate on region $(\mathrm{M})$. Note that our discussion here includes the special case when region (I) shrinks to zero.

We note that the cylindrical equilibrium relation Eq. (16) presents no additional constraint at $r_{1}$. We next focus our attention on the expression for the Shafranov shift Eq. (27) evaluated at $r_{1}$

$$
\Delta^{\prime}\left(r_{1}\right)=\frac{1}{R r_{1} f^{(1)^{2}}\left(r_{1}\right)} \int_{r_{0}}^{r_{1}}\left[f^{(1)^{2}}(r)-\frac{2 r p^{(2)^{\prime}}(r)}{B_{0}^{2}}\right] r d r
$$

In order for $\Delta^{\prime}\left(r_{1}\right)$ to be sufficiently well behaved at $r_{1}$ one of the necessary conditions is that

$$
\int_{r_{0}}^{r_{1}} f^{(1)^{2}}(r) r d r=\int_{r_{0}}^{r_{1}} \frac{2 r p^{(2)^{\prime}}(r)}{B_{0}^{2}} r d r
$$

First we note that if $p^{(2)}$ is monotonically decreasing with $r$, the constraint Eq. (31) cannot be satisfied; $\Delta^{\prime}\left(r_{1}\right)$ will become infinite. Therefore, instead of the normal decreasing pressure profile throughout, a necessary condition is that we have to arrange the pressure to increase somewhere before reaching the poloidal field reversal layer. Next, we show that there are profiles which allow $\Delta^{\prime}\left(r_{1}\right)$ to remain finite. We utilize Eq. (16) to rewrite Eq. (30) as

$$
\Delta^{\prime}\left(r_{1}\right)=\frac{1}{R r_{1} f^{(1)^{2}}\left(r_{1}\right)}\left\{\left(r_{1} f^{(1)}\right)^{2}+\int_{r_{0}}^{r_{1}}\left[f^{(1)^{2}}(r)+2 r g^{(2)^{\prime}}\right] r d r\right\}
$$

In Eq. (32), we have used the condition that $f^{(1)}\left(r_{0}\right)=0$. Now if we let $g^{(2)^{\prime}}=f^{(1)} f^{(1)^{\prime}} / 2$, then Eq. (32) can be integrated to give $\Delta^{\prime}\left(r_{1}\right)=3 r_{1} / 2 R$. Because this is an integral constraint, if this were the only constraint, the equilibrium could still be constructed quite robustly. Next we need to look at the ellipticity equation. This equation is singular at both $r_{0}$ and $r_{1}$. Therefore, no boundary condition can be imposed on its solution. Both of the values $E\left(r_{0}\right)$ and $E\left(r_{1}\right)$ are determined by the equation itself. The matching at $r=r_{0}$ can be done in the same fashion as in the previous subsection. But the matching at $r=r_{1}$ can not be accomplished in general. It is possible that by continuously changing the boundary ellipticity, we could match $E\left(r_{1}\right)$. But these are isolated equilibria. Any slight change in the ellipticity at the boundary will violate the equilibrium condition. They are therefore not 
experimentally realizable. Similar considerations can be applied to the flux renormalization condition and the higher order shape functions. Thus, we have constructed a mathematical proof that negative current cannot be driven in the central region. 


\section{NUMERICAL EXAMPLE OF AN EQUILIBRIUM WITH A CURRENT HOLE}

In this section, we give a numerical example of the profiles of an equilibrium with a current hole. In this example, for the minor radius we use $a=1$, for the major radius we use $R=3$, and we let the edge $q$ value be $q_{a}=3$. The toroidal magnetic field $B_{0}$ is taken to be 1 . The current profile is assumed to be

$$
j=\left\{\begin{array}{lll}
0 & \text { for } & r \leq r_{0} \\
j_{0} & \text { for } & \mathrm{r}_{0} \leq \mathrm{r} \leq \mathrm{r}_{n} \\
j_{0} \frac{1-r}{1-r_{n}} & & \mathrm{r}_{n} \leq \mathrm{r} \leq 1
\end{array} .\right.
$$

We know that the first order $f^{(1)}(r)$ is given by

$$
f^{(1)}(r)=\frac{\int r j(r) d r}{r}
$$

and the value $j_{0}$ is determined from

$$
q_{a}=\frac{a B_{0}}{R f^{(1)}(a)}
$$

In the example shown, we use $r_{0}=0.3, r_{n}=0.8$ and obtain $j_{0}=0.3072193$. For the pressure gradient we assume

$$
p^{(2)^{\prime}}=\frac{-\beta_{p}}{r} f(r f)^{\prime}
$$

with a constant value of $\beta_{p}=0.5$. Then all the equations can be integrated in a straightforward manner. The current profile $j(r)$ together with $f^{(1)}$ and $f^{(1)^{\prime}}$ are shown in Fig. 2. The $j(r)$ profile is marked with \#, the $f^{(1)}$ marked with $*$ and $f^{(1)^{\prime}}$ marked with + . Note that both $j$ and $f^{(1)^{\prime}}$ have a jump at $r=0.3$. The pressure profile and its gradient $p^{(2)}(r)$, and $p^{(2)^{\prime}}(r)$ are shown in Fig. 3. The $p^{(2)}$ is marked with + , and $p^{(2)^{\prime}}$ marked with $*$. Note that $p^{(2)}$ is smoothly joined at $r=0.3$ with its derivative 0 there. Becasue we have taken the $\beta_{p}$ value to be 0.5 , Fig. 3 is also the plot for $g^{(2)}(r), g^{(2)^{\prime}}(r)$. Finally, the profiles of $\Delta(r), \Delta^{\prime}(r)$, and $E(r)$ are shown in Fig. 4. The $E(r)$ profile is marked with \#, the $\Delta(r)$ marked with + , and $\Delta^{\prime}(r)$ marked with $*$. The boundary conditions used in generating these curves are 
$E(1)=0.1$ and $\Delta\left(r_{0}\right)=0$. It is seen that all these quantities are well defined. Note also that the derivatives of these quantities, like the current density, have a contact or jump discontinuity at the hole edge.

Work is underway on numerically calculated current-hole equilibria with non-circular boundary shapes. On physical grounds, the surface functions $p^{\prime}$ and $g g^{\prime}$ must be zero inside a current hole. This suggests a possible approach where we choose well-behaved surface functions $p^{\prime}$ and $g g^{\prime}$ with the only restriction being that these functions go to zero smoothly at the minimum value of $f=0$ in the computational domain. (In regular equilibria at least one of these functions must be non-zero at $f=f_{\min }=0$ to keep the on-axis current density finite.) We anticipate that equilibria with a central hole should develop in the iteration process because the alternative, namely a magnetic axis with zero on-axis current density, ${ }^{5}$ is not probable. 


\section{CONCLUSIONS}

In this paper, we have revisited the theory of Greene, Johnson and Weimer and extended their theory to include equilibria with a central current hole (a region without toroidal or poloidal current). We show that this type of equilibrium consists of a central region with constant pressure and no poloidal magnetic field. It is joined through a contact discontinuity with the outer region with a regular tokamak behavior. At this discontinuity, all the geometric quantities such as Shafranov shift and ellipticity are continous, but discontinuity of their derivatives could exist. The equations that determine equilibria in the current hole are less singular than near the magnetic axis. All the physical quantities exist and are finite. In particular, these include the case of a current jump at the current hole boundary. The experimentally observed current hole states do not have to be transient states and can be in true magnetohydrodynamic equilibrium. We have also analyzed the possibility of a negative current region in between the current hole and the external positive current region. This type of equilibrium has been found to be nonexistent in general. Only isolated solutions which do not depend on the boundary and profile continuously are possible. They do not

correspond to experimentally observable states. Our results agree with the experimental observation that current hole equilibria have been found to persist, while no negative currents have ever been observed in these experiments. 


\section{ACKNOWLEDGMENTS}

This research is supported by the U.S. Department of Energy under Grant No. DEFG03-95ER54309. The authors would like to acknowledge interesting discussions with Drs. S.C. Jardin and T.H. Jensen. They would also like to thank Dr. M.S. Chance for carefully reading through the manuscript and Dr. S.C. Jardin for pointing out the work of Stratton et al. ${ }^{7}$ Lastly, we acknowledge the referee's meticulous suggestions on the grammer and style of this paper. 


\section{REFERENCES}

${ }^{1}$ T.S. Taylor, D.A. Humphreys, L.L. Lao, B.W. Rice, R. Wolf, Bull. Am. Phys. Soc. 43, 1763 (1998).

${ }^{2}$ N.C. Hawkes, B.C. Stratton, T. Tala, et al., Phys. Rev. Lett. 87, 115001 (2001).

3 T. Fujita, T. Oikawa, T. Suzuki, et al., Phys. Rev. Lett. 87, 245001 (2001).

${ }^{4}$ J.M. Greene, J.L. Johnson, K. Weimer, Phys. Fluids 14, 671 (1971).

${ }^{5}$ T.H. Jensen, R.L. Miller, and Y.R. Lin-Liu, Phys. Plasmas 3, 1656 (1996).

${ }^{6}$ G.T.A Huysmans, T.C. Hender, N.C. Hawkes, and X. Litaudon Phys. Rev. Lett. 87, 245002 (2001).

${ }^{7}$ B.C. Stratton, J.A. Breslau, R.V. Budny, S.C. Jardin, W. Park, H.R. Strauss, L.E. Zakharov, B. Alper, V. Drozdov, N.C. Hawkes, S. Reyes-Cortes, and Contributors to the EFDA-JET Work Programme, Plasma Phys. and Control. Fusion 44, 1127 (2002).

${ }^{8}$ T. Oikawa, Private communications (2002). 


\section{List of Figure Captions}

Fig. 1. Coordinate systems.

Fig. 2. Profiles for current $j(r)$ and $f^{(1)}$ and $f^{(1)^{\prime}}$. The $j(r)$ profile is marked with \#, the $f^{(1)}$ marked with $*$ and $f^{(1)^{\prime}}$ marked with + . Note that both $j$ and $f^{(1)^{\prime}}$ has a jump at the hole edge $r=0.3$.

Fig. 3. Profiles for the pressure $p^{(2)}(r)$, and its gradient $p^{(2)^{\prime}}(r)$. The $p^{(2)}$ is marked with + , and $p^{(2)^{\prime}}$ marked with $*$. Note that $p^{(2)}$ is smoothly joined at $r=0.3$ with its derivative 0 there. Becasue we have taken the $\beta_{p}$ value to be 0.5 , this figure is also the plot for $g^{(2)}(r)$ and $g^{(2)^{\prime}}(r)$.

Fig. 4. Profiles of $\Delta(r), \Delta^{\prime}(r)$, and $E(r)$. The $E(r)$ profile is marked with \#, the $\Delta(r)$ marked with + , and $\Delta^{\prime}(r)$ marked with $*$. The boundary conditions used in generating these curves are $E(1)=0.1$ and $\Delta\left(r_{0}\right)=0$. 


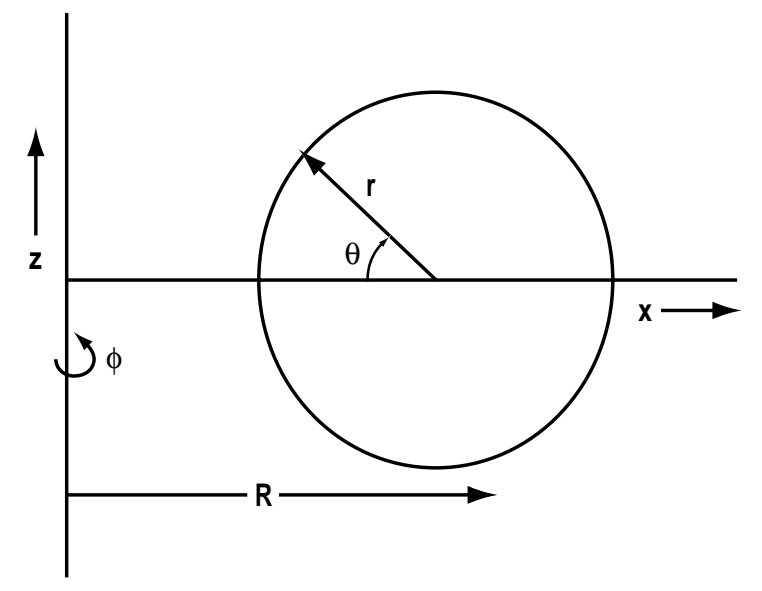

M.S. Chu Figure 1 


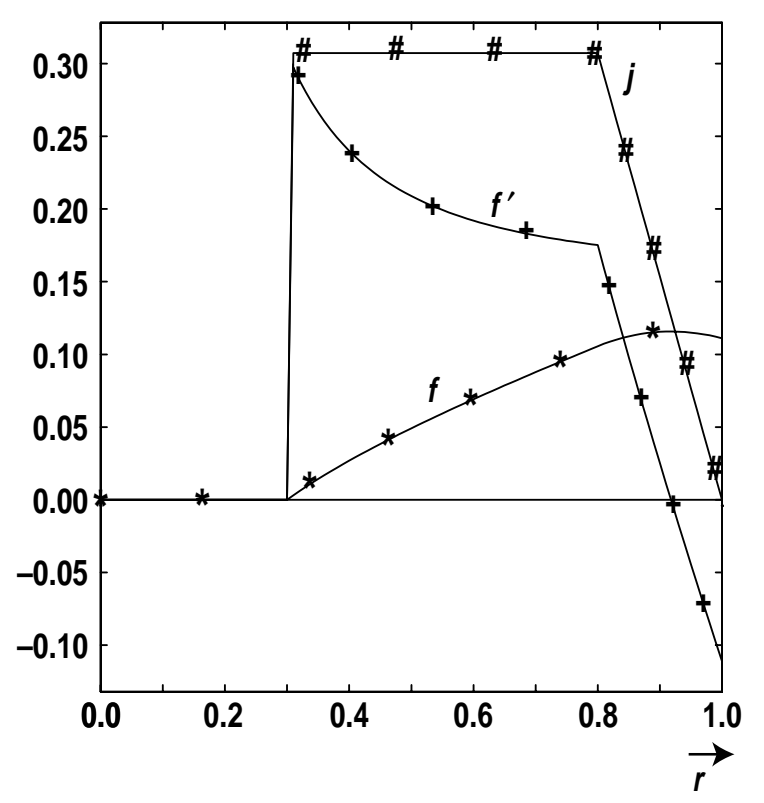

M.S. Chu Figure 2 


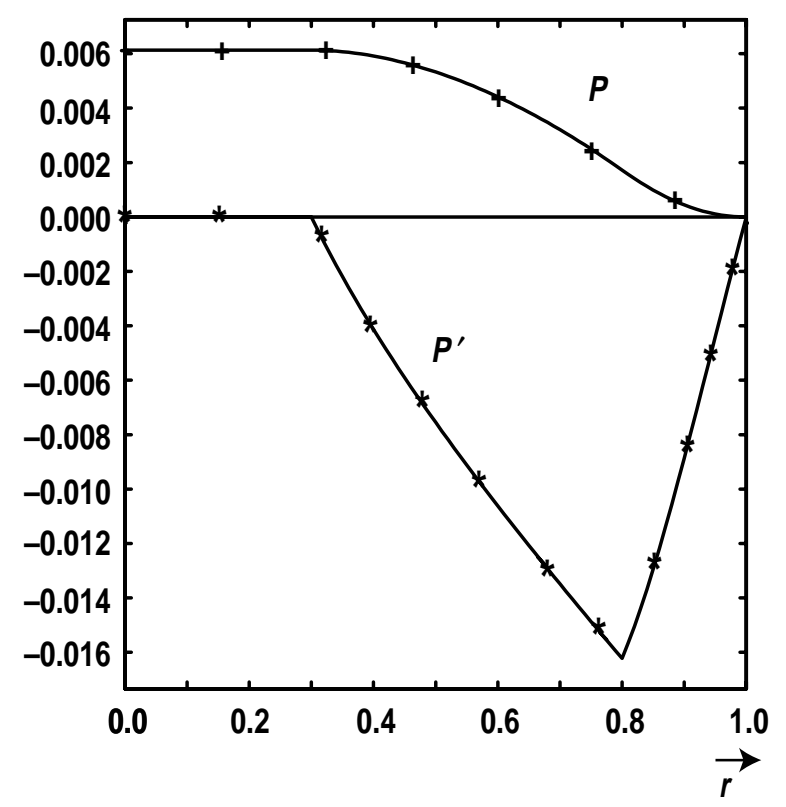

M.S. Chu Figure 3 


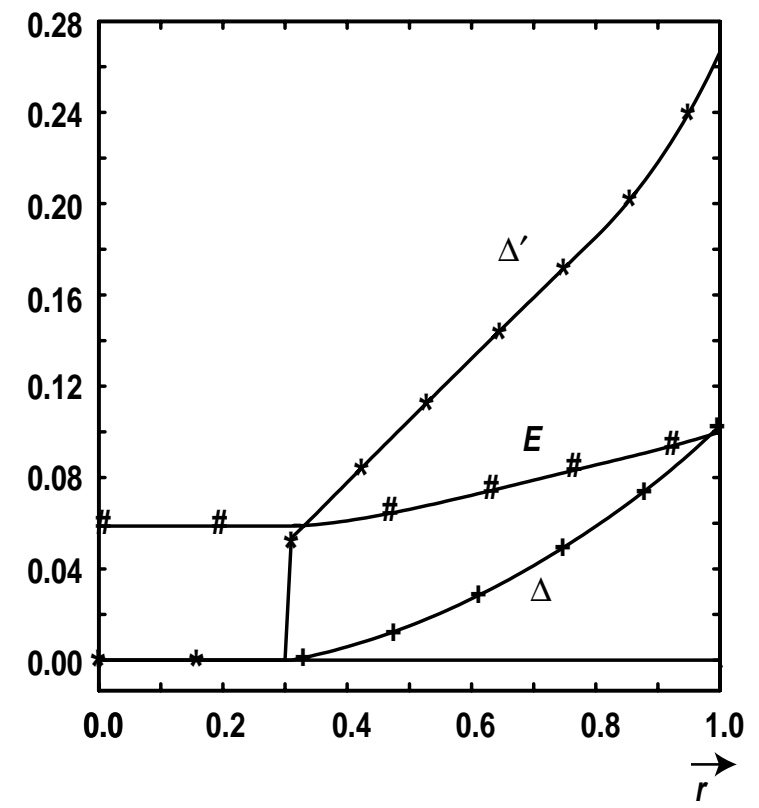

M.S. Chu Figure 4 